\title{
Field-Induced Quantum Critical Point and Nodal Superconductivity in the Heavy-Fermion Superconductor $\mathrm{Ce}_{2} \mathrm{PdIn}_{8}$
}

\author{
J. K. Dong, ${ }^{1}$ H. Zhang, ${ }^{1}$ X. Qiu, ${ }^{1}$ B. Y. Pan, ${ }^{1}$ Y. F. Dai, ${ }^{1}$ T. Y. Guan, ${ }^{1}$ S. Y. Zhou, ${ }^{1}$ \\ D. Gnida, ${ }^{2}$ D. Kaczorowski, ${ }^{2}$ and S. Y. $\mathrm{Li}^{1, *}$ \\ ${ }^{1}$ Department of Physics, State Key Laboratory of Surface Physics, and Laboratory of Advanced Materials, \\ Fudan University, Shanghai 200433, China \\ ${ }^{2}$ Institute of Low Temperature and Structure Research, Polish Academy of Sciences, P.O. Box 1410, 50-950 Wroclaw, Poland
}

(Received 3 August 2010; revised manuscript received 18 July 2011; published 15 September 2011)

The in-plane resistivity $\rho$ and thermal conductivity $\kappa$ of the heavy-fermion superconductor $\mathrm{Ce}_{2} \mathrm{PdIn}_{8}$ single crystals were measured down to $50 \mathrm{mK}$. A field-induced quantum critical point, occurring at the upper critical field $H_{c 2}$, is demonstrated from the $\rho(T) \sim T$ near $H_{c 2}$ and $\rho(T) \sim T^{2}$ when further increasing the field. The large residual linear term $\kappa_{0} / T$ at zero field and the rapid increase of $\kappa(H) / T$ at low field give evidence for nodal superconductivity in $\mathrm{Ce}_{2} \mathrm{PdIn}_{8}$. The jump of $\kappa(H) / T$ near $H_{c 2}$ suggests a first-order-like phase transition at low temperature. These results mimic the features of the famous CeCoIn ${ }_{5}$ superconductor, implying that $\mathrm{Ce}_{2} \mathrm{PdIn}_{8}$ may be another interesting compound to investigate for the interplay between magnetism and superconductivity.

DOI: 10.1103/PhysRevX.1.011010

The interplay between magnetism and superconductivity has been a central issue for heavy-fermion superconductors [1], high- $T_{c}$ cuprates [2], and iron pnictides [3]. Among them, one particularly interesting case is the heavy-fermion superconductor $\mathrm{CeCoIn}_{5}$, with $T_{c}=2.3 \mathrm{~K}$ at ambient pressure [4]. Its superconducting gap has $d$-wave symmetry [5,6]. While there is no static magnetism in $\mathrm{CeCoIn}_{5}$ at zero field, a field-induced antiferromagnetic (AF) quantum critical point (QCP) has been clearly demonstrated by resistivity and specific heat measurements $[7,8]$. Initially, it was very puzzling why the AF QCP is located right at the upper critical field $H_{c 2}$.

Meanwhile, the observations of first-order phase transition at low temperature and $H_{c 2}$ and a second magnetization and specific heat anomaly well inside the superconducting state have been interpreted as the signature of a Fulde-Ferrell-Larkin-Ovchinnikov (FFLO) superconducting state [5,9-12]. The novel FFLO state with broken spatial symmetry was predicted in the 1960s $[13,14]$, but it has never been experimentally verified before. The possible FFLO state at the low-temperaturehigh-field (LTHF) corner of the $H-T$ phase diagram of $\mathrm{CeCoIn}_{5}$ has stimulated extensive studies [15].

More recently, NMR, neutron scattering, and muon spin rotation ( $\mu \mathrm{SR})$ experiments have provided clear evidence for a field-induced magnetism in this LTHF part of the phase diagram [16-21]. It was identified as a spin-density wave (SDW) order with an incommensurate modulation

\footnotetext{
*shiyan_li@fudan.edu.cn

Published by the American Physical Society under the terms of the Creative Commons Attribution 3.0 License. Further distribution of this work must maintain attribution to the author(s) and the published article's title, journal citation, and DOI.
}

$\mathbf{Q}=(0.44,0.44,0.5)$. Interestingly, this SDW order disappears in the normal state above $H_{c 2}$, showing that magnetic order and superconductivity in $\mathrm{CeCoIn}_{5}$ are directly coupled [16,17]. While this has nicely explained the field-induced AF QCP at $H_{c 2}[7,8]$, the physical origin of this LTHF superconducting $Q$ phase is still under debate. For example, Yanase and Sigrist have suggested that the incommensurate SDW order is stabilized in the FFLO state by the appearance of the Andreev bound state localized around the zeros of the FFLO order parameter [22]. Aperis, Varelogiannis, and Littlewood have argued that the $Q$ phase is a pattern of coexisting condensates: a $d$-wave singlet superconducting state, a staggered $\pi$-triplet superconducting state, and an SDW [23]. In this latter scenario, the FFLO state is excluded. Therefore, it is still unclear whether the $Q$ phase relates to an FFLO state.

To clarify this important issue, it will be very helpful to find more compounds with this kind of $Q$ phase. However, none of the related compounds, ${\mathrm{Ce} M \mathrm{In}_{5}}_{5}(M=\mathrm{Rh}$, Ir) and $\mathrm{Ce}_{2} M \mathrm{In}_{8}(M=\mathrm{Co}, \mathrm{Rh}, \mathrm{Ir})$, shows any sign of the $Q$ phase [24-28]. Recently, it was found that $\mathrm{Ce}_{2} \mathrm{PdIn}_{8}$ is also a heavy-fermion superconductor with $T_{c}=0.68 \mathrm{~K}$ at ambient pressure [29]. In this paper, we report the observations of a field-induced QCP, the nodal superconductivity, and a first-order-like phase transition at $H_{c 2}$ in $\mathrm{Ce}_{2} \mathrm{PdIn}_{8}$ by resistivity and thermal conductivity measurements. Our findings suggest that $\mathrm{Ce}_{2} \mathrm{PdIn}_{8}$ may also have an exotic superconducting state as in $\mathrm{CeCoIn}_{5}$.

Single crystals of $\mathrm{Ce}_{2} \mathrm{PdIn}_{8}$ were grown from indium flux, as described for $\mathrm{Ce}_{2} \mathrm{CoIn}_{8}$ [26]. It can be viewed as a periodic stacking of two layers of $\mathrm{CeIn}_{3}$ on a layer of $\mathrm{PdIn}_{2}$. To avoid the impurity phase of $\mathrm{CeIn}_{3}$ [30], very thin crystals were selected for this study, with the typical thickness $40 \mu \mathrm{m}$. Contacts were made with soldered indium, which were used for both resistivity and thermal conductivity 

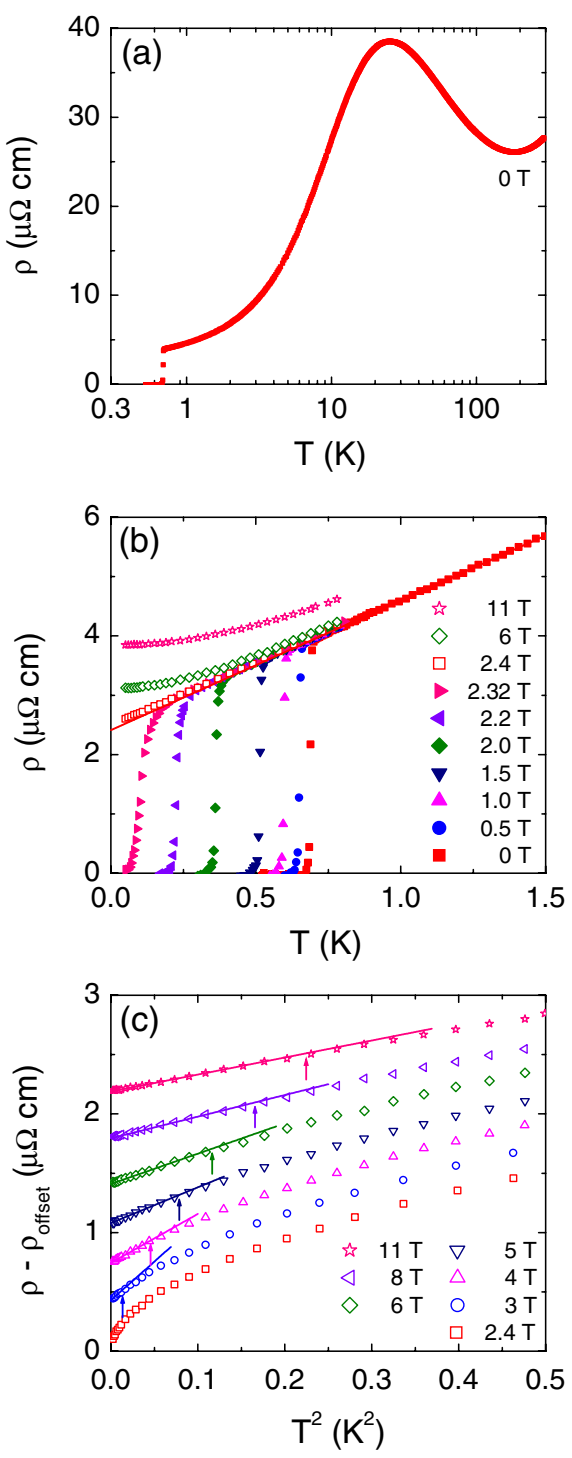

FIG. 1. (a) In-plane resistivity of the $\mathrm{Ce}_{2} \mathrm{PdIn}_{8}$ single crystal in zero field. The curve is very smooth below $20 \mathrm{~K}$, showing no impurity phases in the sample. (b) Low-temperature resistivity of $\mathrm{Ce}_{2} \mathrm{PdIn}_{8}$ in magnetic fields applied along the $c$ axis. The solid line is a linear fit of the zero-field data between 0.7 and $1.5 \mathrm{~K}$. (c) $\rho$ vs $T^{2}$ for $H=2.4,3,4,5,6,8$, and $11 \mathrm{~T}$. (Data sets are offset for clarity.) The solid lines are fits to $\rho=\rho_{0}+A T^{2}$. The arrows indicate the upper limit of the temperature range of $T^{2}$ behavior.

measurements. The resulting contact resistance is $\sim 5 \mathrm{~m} \Omega$ at $1.5 \mathrm{~K}$. In-plane thermal conductivity was measured in a dilution refrigerator, using a standard four-wire steady-state method with two $\mathrm{RuO}_{2}$ chip thermometers, calibrated in situ against a reference $\mathrm{RuO}_{2}$ thermometer. Magnetic fields were applied along the $c$ axis and perpendicular to the heat current. To ensure a homogeneous field distribution in the sample, all fields were applied at temperature above $T_{c}$.

Figure 1(a) shows the in-plane resistivity of our $\mathrm{Ce}_{2} \mathrm{PdIn}_{8}$ single crystal in zero field. A broad peak is found at $T_{\text {coh }}=25 \mathrm{~K}$, which is attributed to a crossover between

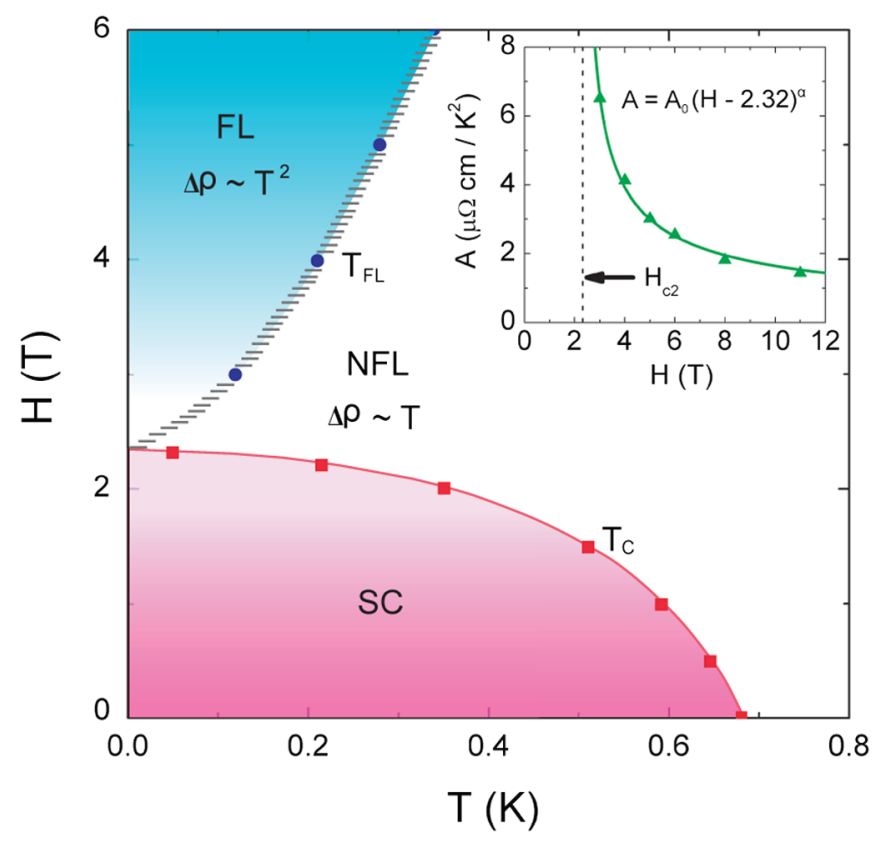

FIG. 2. The $H-T$ phase diagram of $\mathrm{Ce}_{2} \mathrm{PdIn}_{8}$ determined from the resistivity measurements. The $T_{c}$ is defined at $10 \%$ of the normal-state resistivity in each field. The $T_{F L}$ is defined as the upper limit of the temperature range of $T^{2}$-dependence Fermi liquid behavior. This phase diagram suggests a field-induced quantum critical point located at $H_{c 2}=2.32 \mathrm{~T}$. The inset shows the field dependence of the coefficient $A$ of $\rho=\rho_{0}+A T^{2}$, which tends to diverge toward $H_{c 2}=2.32 \mathrm{~T}$.

incoherent scattering at high temperature and the development of a coherent heavy-fermion state at lower temperatures. The curve is very smooth below $20 \mathrm{~K}$, suggesting that there are no impurity phases, especially for the antiferromagnetic $\mathrm{CeIn}_{3}$ with $T_{N} \sim 10 \mathrm{~K}[29,30]$. In Fig. 1(b), the low-temperature $\rho(T)$ in magnetic fields $H \| c$ up to $11 \mathrm{~T}$ are plotted. From the zero-field data, $T_{c}=0.68 \mathrm{~K}$ is obtained, which is defined at the value of $10 \%$ of the normal state. The 10-90\% transition width is only $20 \mathrm{mK}$. It is found that $\rho(T)$ obeys $T$-linear dependence nicely above $T_{c}=0.68 \mathrm{~K}$, up to about $2 \mathrm{~K}$. A linear fit of the data between 0.7 and $1.5 \mathrm{~K}$ gives a residual resistivity $\rho_{0}=2.41 \mu \Omega \mathrm{cm}$.

With increasing field, $T_{c}$ gradually decreases, to $50 \mathrm{mK}$ at $H=2.32 \mathrm{~T}$. This field is determined as the bulk $H_{c 2}$ for $H \| c$. In slightly higher field $H=2.4 \mathrm{~T}$, the resistive transition is completely suppressed, and the $T$-linear behavior of normal-state $\rho(T)$ persists all the way down to $50 \mathrm{mK}$. In $H>2.4 \mathrm{~T}$, the curves show clear deviation from the $T$-linear dependence. The data of $H=2.4,3,4,5,6,8$, and $11 \mathrm{~T}$ are plotted as $\rho$ vs $T^{2}$ in Fig. 1(c). It is clearly seen that a Fermi liquid behavior of resistivity, $\rho \sim A T^{2}$, develops with increasing field. The inset of Fig. 2 plots the field dependence of the coefficient $A$, which tends to diverge toward $H_{c 2}=2.32 \mathrm{~T}$. The fitting of $A=$ $A_{0}(H-2.32)^{\alpha}$ gives $\alpha=-0.57 \pm 0.02$. Note that, for $\mathrm{CeCoIn}_{5}\left(\rho_{a b}\right.$ in $\left.H \| c\right)$ and $\operatorname{CeRhIn}_{5}\left(\rho_{c}\right.$ in $H \| a b$ and 


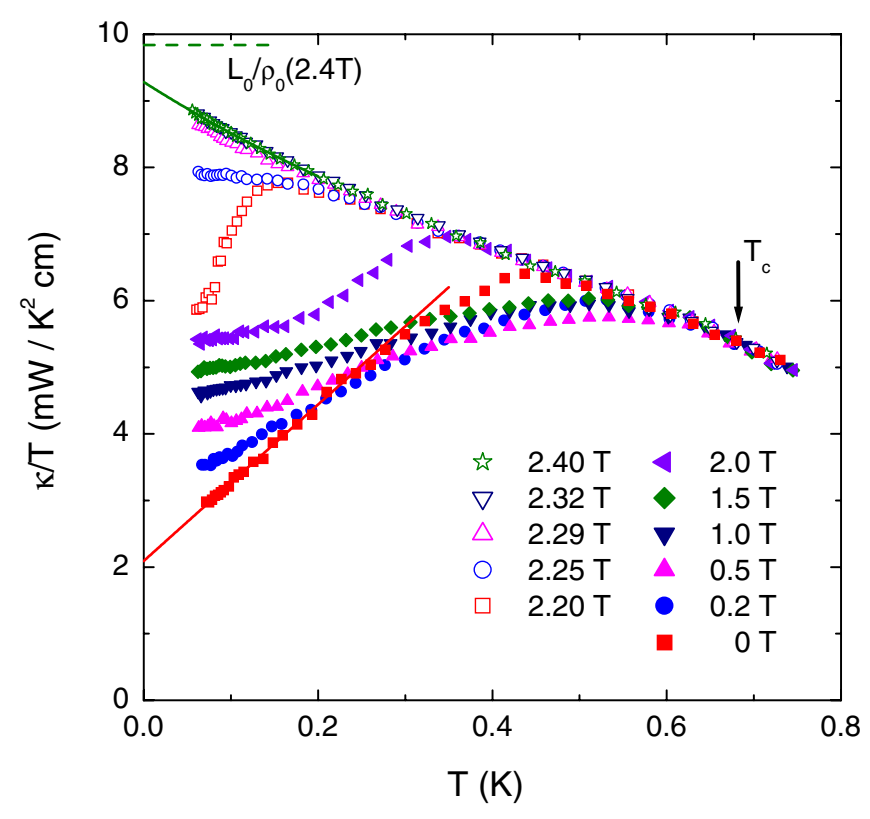

FIG. 3. Low-temperature in-plane thermal conductivity of $\mathrm{Ce}_{2} \mathrm{PdIn}_{8}$ in magnetic fields applied along the $c$ axis. The saturation of $\kappa / T$ toward $2.32 \mathrm{~T}$ confirms it as the bulk $H_{c 2}$. The solid lines are fits to the $H=0$ and $2.4 \mathrm{~T}$ data (see text). The dashed line at the top left of the panel is the normal-state Wiedemann-Franz law expectation $L_{0} / \rho_{0}(2.4 \mathrm{~T})$, with $L_{0}=$ $2.45 \times 10^{-8} \mathrm{~W} \Omega \mathrm{K}^{-2}$ and $\rho_{0}(2.4 \mathrm{~T})=2.49 \mu \Omega \mathrm{cm}$.

under $2.35 \mathrm{GPa}), \alpha=-1.37$ and -0.5 were obtained, respectively $[7,31]$.

Based on these resistivity results, we have constructed an $H-T$ phase diagram for $\mathrm{Ce}_{2} \mathrm{PdIn}_{8}$ in the main panel of Fig. 2. Such a phase diagram is very similar to that of $\mathrm{CeCoIn}_{5}[7,8]$, showing that there is also a field-induced QCP at $H_{c 2}$. To clarify whether it is an AF QCP, more experiments such as NMR, neutron scattering, and $\mu \mathrm{SR}$ are needed to detect the magnetic order below and above $H_{c 2}$, as have been done with $\mathrm{CeCoIn}_{5}$ [16-21].

Next, we turn to the superconducting gap structure of $\mathrm{Ce}_{2} \mathrm{PdIn}_{8}$. Figure 3 shows the temperature dependence of the in-plane thermal conductivity in $H \| c$ up to $2.4 \mathrm{~T}$, plotted as $\kappa / T$ vs $T$. The Wiedemann-Franz (WF) law is first checked in the normal state. The $H=2.4 \mathrm{~T}$ curve is fitted to $\kappa / T=1 /(a+b T)$, since $\rho(2.4 \mathrm{~T})$ is $T$-linear dependent and electrons dominate the heat transport at low temperature. The extrapolation gives $\kappa_{0} / T(2.4 \mathrm{~T})=1 / a \approx$ $9.28 \mathrm{~mW} \mathrm{~K}^{-2} \mathrm{~cm}^{-1}$. This value is about $94 \%$ of the WF law expectation, $\quad L_{0} / \rho_{0}(2.4 \mathrm{~T})=9.84 \mathrm{~mW} \mathrm{~K}^{-2} \mathrm{~cm}^{-1}$, with $L_{0}=2.45 \times 10^{-8} \mathrm{~W} \Omega \mathrm{K}^{-2}$ and $\rho_{0}(2.4 \mathrm{~T})=2.49 \mu \Omega \mathrm{cm}$. The rough satisfaction of the WF law in the normal state shows that our thermal conductivity data are reliable.

In zero field, $\kappa / T$ of $\mathrm{Ce}_{2} \mathrm{PdIn}_{8}$ behaves in a way similar to that of $\mathrm{CeCoIn}_{5}$ [32,33], increasing below $T_{c}$, showing a broad peak at $\sim 0.45 \mathrm{~K}$, and then decreasing toward $T=0$. Below $250 \mathrm{mK}, \kappa / T \propto T$ and extrapolates to $\kappa_{0} / T=2.09 \pm 0.02 \mathrm{mWK}^{-2} \mathrm{~cm}^{-1}$, more than $20 \%$ of

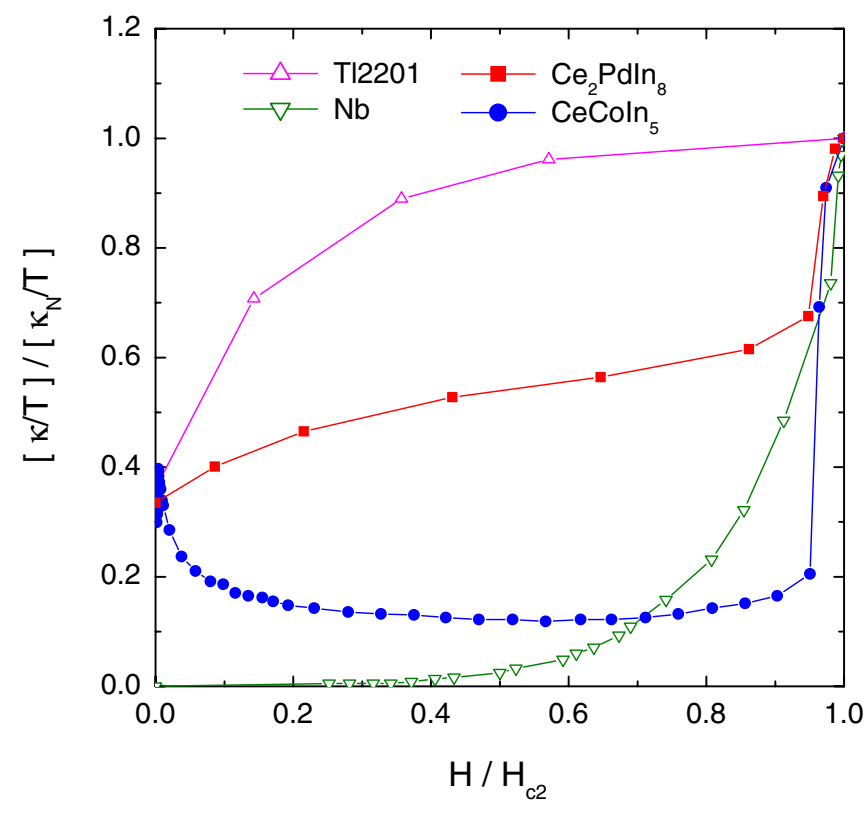

FIG. 4. Normalized $\kappa / T$ to the normal-state value for $\mathrm{Ce}_{2} \mathrm{PdIn}_{8}$ at $60 \mathrm{mK}$, and for $\mathrm{CeCoIn}_{5}$ at $50 \mathrm{mK}$ [33], as a function of $\mathrm{H} / \mathrm{H}_{\mathrm{c2}}$. For both compounds, the sharp jump of $\kappa / T$ near $H_{c 2}$ indicates a first-order phase transition. Similar data of the clean $s$-wave superconductor $\mathrm{Nb}$ [37] and an overdoped $d$-wave superconductor Tl-2201 [38] at $T \rightarrow 0$ are also shown for comparison.

the normal-state value. For the $d$-wave superconductor $\mathrm{CeCoIn}_{5}$, one measurement down to $50 \mathrm{mK}$ obtained $\kappa_{0} / T=17 \mathrm{mWK}^{-2} \mathrm{~cm}^{-1}$ [32], while another measurement down to $10 \mathrm{mK}$ got $\kappa_{0} / T \approx 3 \mathrm{mWK}^{-2} \mathrm{~cm}^{-1}$ [33]. The significant $\kappa_{0} / T$ of $\mathrm{Ce}_{2} \mathrm{PdIn}_{8}$ in zero field is strong evidence for nodes in the superconducting gap [34]. Further angle-dependent experiments will be able to show whether this nodal superconductivity is also $d$-wave, as in $\mathrm{CeCoIn}_{5}$ [5,6], CeRhIn 5 under pressure [35], and CeIrIn ${ }_{5}^{\text {[36] }}$.

The field dependence of $\kappa_{0} / T$ may provide further support for the nodes [34]. In Fig. 3, however, the temperature dependence of $\kappa / T$ changes dramatically in fields, and it is hard to extrapolate $\kappa_{0} / T$ reliably for all the curves. Therefore, we plot $\kappa / T$ at $60 \mathrm{mK}$, normalized to its normal-state value (in bulk $H_{c 2}=2.32 \mathrm{~T}$ ), vs $H / H_{c 2}$ in Fig. 4. For comparison, similar data of the clean $s$-wave superconductor $\mathrm{Nb}$ [37] and an overdoped $d$-wave superconductor Tl-2201 [38] at $T \rightarrow 0$ are also shown. At low field $\left(H / H_{c 2}<0.5\right), \kappa(H) / T$ of $\mathrm{Ce}_{2} \mathrm{PdIn}_{8}$ shows downward curvature as in Tl-2201, providing further support for the nodes. In contrast to the $s$-wave superconductor $\mathrm{Nb}$, the rapid increase of $\kappa(H) / T$ at low field in nodal superconductors results from the Volovik effect of the nodal quasiparticles [39].

It is worth pointing out that the $\kappa(H) / T$ of $\mathrm{Ce}_{2} \mathrm{PdIn}_{8}$ at $60 \mathrm{mK}$ is quite different from the abnormal one of $\mathrm{CeCoIn}_{5}$ at $50 \mathrm{mK}$ [33], which is also plotted in Fig. 4. For CeCoIn 5 , the decrease of $\kappa(H) / T$ above $20 \mathrm{mT}$ was attributed to the 
vortex scattering, since the quasiparticle mean free path $l$ in zero field is very large $(\approx 4000 \AA)$ due to the high purity of the sample [33]. However, for $\mathrm{Ce}_{2} \mathrm{PdIn}_{8}, l \approx$ $420 \AA$ has been estimated [29], roughly one order smaller than $\mathrm{CeCoIn}_{5}$. In this case, vortex scattering does not show a significant effect, and $\kappa(H) / T$ of $\mathrm{Ce}_{2} \mathrm{PdIn}_{8}$ behaves more like the typical $d$-wave superconductor Tl-2201 at low field [38].

From Fig. $4, \kappa(H) / T$ of $\mathrm{CeCoIn}_{5}$ shows an abrupt jump near $H_{c 2}$. This is evidence for the first-order transition [5,9-12], which apparently corresponds to the disappearance of the $Q$ phase at $H_{c 2}[17,18]$. For $\mathrm{Ce}_{2} \mathrm{PdIn}_{8}$, a less sharp jump of $\kappa(H) / T$ is also found near $H_{c 2}$, which hints that the superconducting-to-normal-state transition at very low temperature is first-order-like. This result, together with the field-induced QCP at $H_{c 2}$ and the nodal superconductivity, all mimic those features of $\mathrm{CeCoIn}_{5}$. Therefore, it is possible that there may exist an exotic superconducting state at the LTHF corner of the phase diagram of $\mathrm{Ce}_{2} \mathrm{PdIn}_{8}$. It will be very interesting to search for the $Q$ phase and FFLO state in this second compound after $\mathrm{CeCoIn}_{5}$.

Finally, we check the conditions for $\mathrm{Ce}_{2} \mathrm{PdIn}_{8}$ to form an FFLO state. There are two requirements for the formation of an FFLO state: a very large Maki parameter $(\alpha>1.8)$ and a very clean compound $(\xi \ll l)$ [15]. The Maki parameter $\alpha=\sqrt{2} H_{\text {orb }} / H_{P}$, where $H_{\text {orb }}$ and $H_{P}$ are the orbital and Pauli limiting $H_{c 2}$, reflects the relative strength of the Pauli paramagnetic effect and the orbital effect when a magnetic field is suppressing the superconductivity [40]. For $\mathrm{CeCoIn}_{5}, \alpha=4.6$ and 5.0 were obtained in $H \| a b$ and $H \| c$, respectively [41]. For our $\mathrm{Ce}_{2} \mathrm{PdIn}_{8}$ in $H \| c, H_{\text {orb }}=$ $-0.7 T_{c} d H_{c 2} /\left.d T\right|_{T=T_{c}} \approx 6.8 \mathrm{~T}$ is obtained from the initial slope $d H_{c 2} /\left.d T\right|_{T=T_{c}}=-14.3 \mathrm{~T} / \mathrm{K}$ in Fig. 2. By assuming $H_{P} \approx H_{c 2}(0)=2.32 \mathrm{~T}$, we get $\alpha \approx 2.9$. Therefore, both $\mathrm{CeCoIn}_{5}$ and $\mathrm{Ce}_{2} \mathrm{PdIn}_{8}$ meet the required minimum value of $\alpha$. The in-plane superconducting coherence length $\xi=47-50 \AA$ was estimated for $\mathrm{CeCoIn}_{5}$ [42]. This value is about 100 times smaller than the mean free path $l \approx$ $4000 \AA$ [33], showing that $\mathrm{CeCoIn}_{5}$ is in the clean limit $\xi \ll l$. For $\mathrm{Ce}_{2} \mathrm{PdIn}_{8}, \xi \approx 82 \AA$ and $l \approx 420 \AA$ were estimated [29]. This shows that $\mathrm{Ce}_{2} \mathrm{PdIn}_{8}$ is a clean compound $(\xi<l)$ too, although not as clean as $\mathrm{CeCoIn}_{5}$. Therefore, $\mathrm{Ce}_{2} \mathrm{PdIn}_{8}$ may also fulfill the requirements for the formation of an FFLO state.

In summary, we have measured the resistivity and thermal conductivity of the heavy-fermion superconductor $\mathrm{Ce}_{2} \mathrm{PdIn}_{8}$ single crystal down to $50 \mathrm{mK}$. A field-induced QCP at $H_{c 2}$ is demonstrated by the observation of $\rho \sim T$ near $H_{c 2}$ and the development of $\rho \sim T^{2}$ Fermi liquid behavior at higher fields. The large $\kappa_{0} / T$ at zero field and the rapid increase of $\kappa(H) / T$ at low field give strong evidence for nodal superconductivity. Moreover, the jump of $\kappa / T$ near $H_{c 2}$ indicates a first-order-like phase transition from the superconducting to the normal state at low temperature. These features are similar to those of $\mathrm{CeCoIn}_{5}$, which implies that $\mathrm{Ce}_{2} \mathrm{PdIn}_{8}$ may be another interesting compound to investigate for the interplay between magnetism and superconductivity. The comparison between $\mathrm{CeCoIn}_{5}$ and $\mathrm{Ce}_{2} \mathrm{PdIn}_{8}$ could be valuable for understanding the physical origin of the $Q$ phase and possible FFLO state.

We thank W. Bao, Y. Chen, and Y. Y. Wang for discussions. This work is supported by the National Natural Science Foundation of China, the Ministry of Science and Technology of China (National Basic Research Program No. 2009CB929203), the Program for New Century Excellent Talents in University, Program for Professor of Special Appointment (Eastern Scholar) at Shanghai Institutions of Higher Learning, and the Science and Technology Commission of Shanghai Municipality (STCSM) of China Contract Nos. 08dj1400200 and 08PJ1402100.

[1] Christian Pfleiderer, Superconducting Phases of f-Electron Compounds, Rev. Mod. Phys. 81, 1551 (2009).

[2] P. A. Lee, N. Nagaosa, and X. G. Wen, Doping a Mott Insulator: Physics of High-Temperature Superconductivity, Rev. Mod. Phys. 78, 17 (2006).

[3] D.C. Johnston, The Puzzle of High Temperature Superconductivity in Layered Iron Pnictides and Chalcogenides, Adv. Phys. 59, 803 (2010).

[4] C. Petrovic, P. G. Pagliuso, M. F. Hundley, R. Movshovich, J. L. Sarrao, J. D. Thompson, Z. Fisk, and P. Monthoux, Heavy-Fermion Superconductivity in $\mathrm{CeCoIn}_{5}$ at $2.3 \mathrm{~K}$, J. Phys. Condens. Matter 13, L337 (2001).

[5] K. Izawa, H. Yamaguchi, Yuji Matsuda, H. Shishido, R. Settai, and Y. Onuki, Angular Position of Nodes in the Superconducting Gap of Quasi-2D Heavy-Fermion Superconductor CeCoIn 5 , Phys. Rev. Lett. 87, 057002 (2001).

[6] K. An, T. Sakakibara, R. Settai, Y. Onuki, M. Hiragi, M. Ichioka, and K. Machida, Sign Reversal of Field-Angle Resolved Heat Capacity Oscillations in a Heavy Fermion Superconductor $\mathrm{CeCoIn}_{5}$ and $d_{x^{2}-y^{2}}$ Pairing Symmetry, Phys. Rev. Lett. 104, 037002 (2010).

[7] J. Paglione, M. A. Tanatar, D. G. Hawthorn, Etienne Boaknin, R. W. Hill, F. Ronning, M. Sutherland, Louis Taillefer, C. Petrovic, and P.C. Canfield, Field-Induced Quantum Critical Point in $\mathrm{CeCoIn}_{5}$, Phys. Rev. Lett. 91, 246405 (2003).

[8] A. Bianchi, R. Movshovich, I. Vekhter, P. G. Pagliuso, and J.L. Sarrao, Avoided Antiferromagnetic Order and Quantum Critical Point in $\mathrm{CeCoIn}_{5}$, Phys. Rev. Lett. 91, 257001 (2003).

[9] T. P. Murphy, Donavan Hall, E. C. Palm, S. W. Tozer, C. Petrovic, Z. Fisk, R. G. Goodrich, P. G. Pagliuso, J. L. Sarrao, and J.D. Thompson, Anomalous Superconductivity and Field-Induced Magnetism in $\mathrm{CeCoIn}_{5}$, Phys. Rev. B 65, 100514(R) (2002). 
[10] T. Tayama, A. Harita, T. Sakakibara, Y. Haga, H. Shishido, R. Settai, and Y. Onuki, Unconventional Heavy-Fermion Superconductor $\mathrm{CeCoIn}_{5}$ : dc Magnetization Study at Temperatures Down to $50 \mathrm{mK}$, Phys. Rev. B 65, 180504 (R) (2002).

[11] A. Bianchi, R. Movshovich, N. Oeschler, P. Gegenwart, F. Steglich, J. D. Thompson, P. G. Pagliuso, and J. L. Sarrao, First-Order Superconducting Phase Transition in $\mathrm{CeCoIn}_{5}$, Phys. Rev. Lett. 89, 137002 (2002).

[12] A. Bianchi, R. Movshovich, C. Capan, P. G. Pagliuso, and J.L. Sarrao, Possible Fulde-Ferrell-Larkin-Ovchinnikov Superconducting State in $\mathrm{CeCoIn}_{5}$, Phys. Rev. Lett. 91, 187004 (2003).

[13] P. Fulde and R. A. Ferrell, Superconductivity in a Strong Spin-Exchange Field, Phys. Rev. 135, A550 (1964).

[14] A. I. Larkin and Y. N. Ovchinnikov, Inhomogeneous State of Superconductors, Zh. Eksp. Teor. Fiz. 47, 1136 (1964) [Inhomogeneous State of Superconductors, Sov. Phys. JETP 20, 762 (1965)].

[15] Y. Matsuda and H. Shimahara, Fulde-Ferrell-LarkinOvchinnikov State in Heavy Fermion Superconductors, J. Phys. Soc. Jpn. 76, 051005 (2007), and references therein.

[16] B. L. Young, R. R. Urbano, N. J. Curro, J. D. Thompson, J. L. Sarrao, A. B. Vorontsov, and M. J. Graf, Microscopic Evidence for Field-Induced Magnetism in $\mathrm{CeCoIn}_{5}$, Phys. Rev. Lett. 98, 036402 (2007).

[17] M. Kenzelmann, Th. Strässle, C. Niedermayer, M. Sigrist, B. Padmanabhan, M. Zolliker, A.D. Bianchi, R. Movshovich, E. D. Bauer, J.L. Sarrao, and J.D. Thompson, Coupled Superconducting and Magnetic Order in $\mathrm{CeCoIn}_{5}$, Science 321, 1652 (2008).

[18] J. Spehling, R. H. Heffner, J. E. Sonier, N. Curro, C. H. Wang, B. Hitti, G. Morris, E. D. Bauer, J. L. Sarrao, F. J. Litterst, and H.-H. Klauss, Field-Induced Coupled Superconductivity and Spin Density Wave Order in the

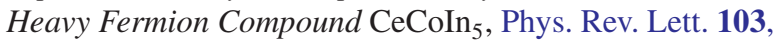
237003 (2009).

[19] G. Koutroulakis, M. D. Stewart, Jr., V.F. Mitrović, M. Horvatić, C. Berthier, G. Lapertot, and J. Flouquet, Field Evolution of Coexisting Superconducting and Magnetic Orders in $\mathrm{CeCoIn}_{5}$, Phys. Rev. Lett. 104, 087001 (2010).

[20] M. Kenzelmann, S. Gerber, N. Egetenmeyer, J. L. Gavilano, Th. Strässle, A. D. Bianchi, E. Ressouche, R. Movshovich, E.D. Bauer, J.L. Sarrao, and J.D. Thompson, Evidence for a Magnetically Driven Superconducting $Q$ Phase of $\mathrm{CeCoIn}_{5}$, Phys. Rev. Lett. 104, 127001 (2010).

[21] K. Kumagai, H. Shishido, T. Shibauchi, and Y. Matsuda, Evolution of Paramagnetic Quasiparticle Excitations Emerged in the High-Field Superconducting Phase of $\mathrm{CeCoIn}_{5}$, Phys. Rev. Lett. 106, 137004 (2011).

[22] Y. Yanase and M. Sigrist, Antiferromagnetic Order and $\pi$-Triplet Pairing in the Fulde-Ferrell-LarkinOvchinnikov State, J. Phys. Soc. Jpn. 78, 114715 (2009).

[23] A. Aperis, G. Varelogiannis, and P. B. Littlewood, Magnetic-Field-Induced Pattern of Coexisting Condensates in the Superconducting State of $\mathrm{CeCoIn}_{5}$, Phys. Rev. Lett. 104, 216403 (2010).

[24] T. Park, F. Ronning, H. Q. Yuan, M. B. Salamon, R. Movshovich, J. L. Sarrao, and J. D. Thompson, Hidden
Magnetism and Quantum Criticality in the Heavy Fermion Superconductor $\mathrm{CeRhIn}_{5}$, Nature (London) 440, 65 (2006).

[25] C. Capan, L. Balicas, T.P. Murphy, E. C. Palm, R. Movshovich, D. Hall, S. W. Tozer, M. F. Hundley, E. D. Bauer, J.D. Thompson, J.L. Sarrao et al., Unusual Metamagnetism in CeIrIn ${ }_{5}$, Phys. Rev. B 80, 094518 (2009).

[26] G. Chen, S. Ohara, M. Hedo, Y. Uwatoko, K. Saito, M. Sorai, and I. Sakamoto, Observation of Superconductivity in Heavy-Fermion Compounds of $\mathrm{Ce}_{2} \mathrm{CoIn}_{8}$, J. Phys. Soc. Jpn. 71, 2836 (2002).

[27] M. Nicklas, V. A. Sidorov, H. A. Borges, P. G. Pagliuso, C. Petrovic, Z. Fisk, J.L. Sarrao, and J.D. Thompson, Magnetism and Superconductivity in $\mathrm{Ce}_{2} \mathrm{RhIn}_{8}$, Phys. Rev. B 67, 020506 (2003).

[28] J. D. Thompson, R. Movshovich, Z. Fisk, F. Bouquet, N. J. Curro, R. A. Fisher, P. C. Hammel, H. Hegger, M. F. Hundley, M. Jaime et al., Superconductivity and Magnetism in a New Class of Heavy-Fermion Materials, J. Magn. Magn. Mater. 226-230, 5 (2001).

[29] D. Kaczorowski, A. P. Pikul, D. Gnida, and V.H. Tran, Emergence of a Superconducting State from an Antiferromagnetic Phase in Single Crystals of the Heavy Fermion Compound $\mathrm{Ce}_{2} \mathrm{PdIn}_{8}$, Phys. Rev. Lett. 103, 027003 (2009).

[30] D. Kaczorowski, A. P. Pikul, D. Gnida, and V.H. Tran, Reply, Phys. Rev. Lett. 104, 059702 (2010).

[31] T. Park, Y. Tokiwa, F. Ronning, H. Lee, E. D. Bauer, R. Movshovich, and J.D. Thompson, Field-Induced Quantum Critical Point in the Pressure-Induced Superconductor CeRhIn ${ }_{5}$, Phys. Status Solidi B 247, 553 (2010).

[32] M. A. Tanatar, Johnpierre Paglione, S. Nakatsuji, D. G. Hawthorn, E. Boaknin, R. W. Hill, F. Ronning, M. Sutherland, Louis Taillefer, C. Petrovic, P. C. Canfield, and Z. Fisk, Unpaired Electrons in the Heavy-Fermion Superconductor $\mathrm{CeCoIn}_{5}$, Phys. Rev. Lett. 95, 067002 (2005).

[33] G. Seyfarth, J. P. Brison, G. Knebel, D. Aoki, G. Lapertot, and J. Flouquet, Multigap Superconductivity in the HeavyFermion System $\mathrm{CeCoIn}_{5}$, Phys. Rev. Lett. 101, 046401 (2008).

[34] H. Shakeripour, C. Petrovic, L. Taillefer, Heat Transport as a Probe of Superconducting Gap Structure, New J. Phys. 11, 055065 (2009).

[35] T. Park, E. D. Bauer, and J.D. Thompson, Probing the Nodal Gap in the Pressure-Induced Heavy Fermion Superconductor CeRhIn ${ }_{5}$, Phys. Rev. Lett. 101, 177002 (2008).

[36] Y. Kasahara, T. Iwasawa, Y. Shimizu, H. Shishido, T. Shibauchi, I. Vekhter, and Y. Matsuda, Thermal Conductivity Evidence for a $d_{x^{2}-y^{2}}$ Pairing Symmetry in the Heavy-Fermion CeIrIn 5 Superconductor, Phys. Rev. Lett. 100, 207003 (2008).

[37] J. Lowell and J. Sousa, Mixed-State Thermal Conductivity of Type II Superconductors, J. Low Temp. Phys. 3, 65 (1970).

[38] C. Proust, E. Boaknin, R. W. Hill, Louis Taillefer, and A.P. Mackenzie, Heat Transport in a Strongly Overdoped Cuprate: Fermi Liquid and a Pure 
d-Wave BCS Superconductor, Phys. Rev. Lett. 89, 147003 (2002).

[39] G. E. Volovik, Superconductivity with Lines of Gap Nodes: Density of States in the Vortex, JETP Lett. 58, 469 (1993) [http://www.jetpletters.ac.ru/ps/1189/article_ 17954.shtml].

[40] K. Maki, Effect of Pauli Paramagnetism on Magnetic Properties of High-Field Superconductors, Phys. Rev. 148, 362 (1966).
[41] K. Kumagai, M. Saitoh, T. Oyaizu, Y. Furukawa, S. Takashima, M. Nohara, H. Takagi, and Y. Matsuda, Fulde-Ferrell-Larkin-Ovchinnikov State in a Perpendicular Field of Quasi-Two-Dimensional CeCoIn ${ }_{5}$, Phys. Rev. Lett. 97, 227002 (2006).

[42] L. DeBeer-Schmitt, C. D. Dewhurst, B. W. Hoogenboom, C. Petrovic, and M. R. Eskildsen, Field Dependent Coherence Length in the Superclean, High- $\kappa$ Superconductor $\mathrm{CeCoIn}_{5}$, Phys. Rev. Lett. 97, 127001 (2006). 